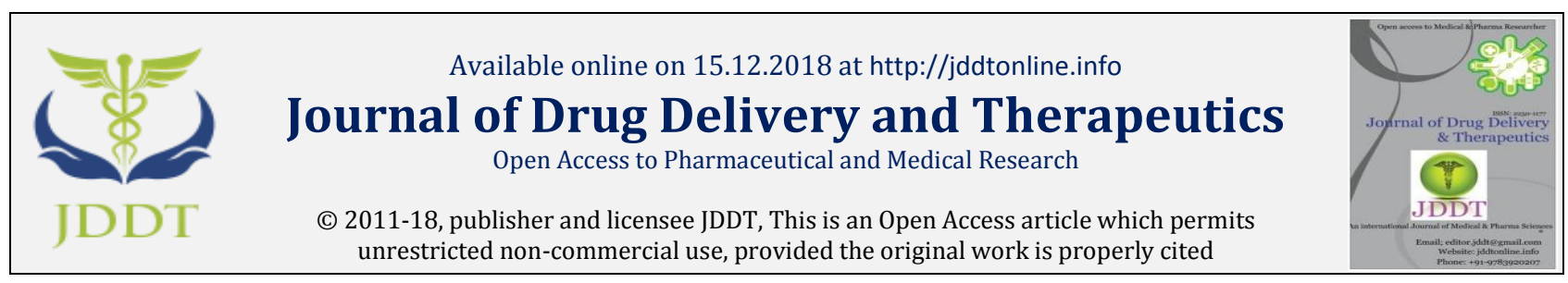

Open $\overbrace{\text { Access }}$

Review Article

\title{
Unique Structures, Properties and Applications of Dendrimers
}

\author{
Ankita Gupta*, Shaifali Dubey, Mayuri Mishra
}

Maharana Pratap college of Pharmacy, Kanpur, India.

\begin{abstract}
Dendrimers are novel three dimensional, hyperbranched globular nano polymeric architectures. Attractive features like nanoscopic size, narrow polydispersity index and excellent control over molecular structure afford dendrimers with ideal drug delivery ability through encapsulating drugs in their interior or covalently conjugating drugs on their surfaces. The adaptable surface functionalization ability enables covalent conjugation of various targeting molecules onto the surface of dendrimers, thereby allowing for generation of various multifunctional nanodevices for targeted drug delivery applications. Drug delivery researchers are especially enthusiastic about possible utility of dendrimers as drug delivery tool. However, to get the maximum benefits of these novel class macromolecules, a research by collaboration is very much essential. Finally, it is one of the youngest and exciting fields of polymer researches where all branches of science can take part and hence, deserves more intensive attention.
\end{abstract}

Keywords: Dendrimers, Drug Delivery, Targeting, Dual Drug Loading, PAMAM

Article Info: Received 15 Oct, 2018; Review Completed 27 Nov 2018; Accepted 29 Nov 2018; Available online 15 Dec 2018

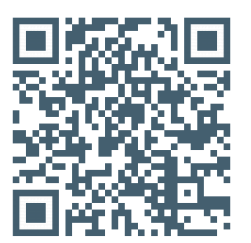

Cite this article as:

Gupta A, Dubey S, Mishra M, Unique Structures, Properties and Applications of Dendrimers, Journal of Drug Delivery and Therapeutics. 2018; 8(6-s):328-339 DOI: http://dx.doi.org/10.22270/jddt.v8i6-s.2083

*Address for Correspondence:

Ms. Ankita Gupta, Asst. Professor, Maharana Pratap College of Pharmacy, Kanpur-209217, India

\section{INTRODUCTION}

The esthetically appealing structures of dendrimers have a stimulating and growing interest in different disciplines of science. These synthetic macromolecules consist of relatively short chains with specific functional groups at the ends. Their structure, which is obtained by means of suitable iterative stepwise reactions sequences, is developed through successive generations and gives rise to monodisperse, highly branched tree-like "dendritic" morphology. Dendrimers are therefore branched, synthetic polymers with layered architectures that show promise in several applications. Up to now, dendrimers have been widely applied in many fields, such as supramolecular chemistry or host-guest chemistry, electrochemistry, photochemistry, nanoparticle synthesis, pollution management, dye decolorization, preparation of monomolecular membranes, curing of epoxy resins, catalysis, drug delivery, and gene transfection ${ }^{1}$. Among them, the use of dendrimers in delivery systems has deserved more attentions in recent years ${ }^{2}$. By regulating dendrimer synthesis, it is possible to precisely manipulate both their molecular weight and chemical composition, thereby allowing predictable tuning of their biocompatibility and pharmacokinetics. Dendrimers offer a unique platform for exploring chemical diversity on the nanoscale. This unique combination of properties makes them ideal candidates for nanotechnology applications in both biological and materials sciences such as use of dendrimers as drugs in their own, dendrimers as drug delivery vehicles or diagnostics 3 .
The potential of dendrimers in treatment of lifestyle and civilization diseases such as cancer 4 , diabetes, viral and microbial infections, Alzheimer etc. has also been recognized 5. Mc Carthy et al. 6 conducted phase 1 human clinical trial of dendrimer-enhanced docetaxel chemotherapeutic product, referred to as DEP ${ }^{\mathrm{TM}}$-Docetaxel The primary objective of the study was to establish the maximum tolerated dose (MTD) and dose limiting toxicities (DLT) of DEP ${ }^{\mathrm{TM}}$-Docetaxel, a new formulation of the major chemotherapeutic agent, docetaxel, which is marketed worldwide under the tradename, Taxotere $\AA$. The preclinical studies of DEP ${ }^{\mathrm{TM}}$-Docetaxel demonstrated the significantly superior anti-cancer effectiveness of the product compared to Taxotere $\AA$ across a range of important cancer types including breast, prostate, lung and ovarian cancer. In addition, $\mathrm{DEP}^{\mathrm{TM}}$-Docetaxel exhibited a lack of the severe toxicity, neutropenia, which is the most important dose-limiting side effect of Taxotere ${ }^{\circledR}$. Use of $\mathrm{DEP}^{\mathrm{TM}}$ technology also improved the water solubility and tissue targeting of docetaxel.

Bioactivity of the designed dendrimers suggests them as promising therapeutic agents in contemporary regenerative medicine, involved in wound healing, tissue and cartilage reconstruction as well as bone mineralization. The potential applications of de novo - designed dendrimers in areas such as boron neutron capture therapy, as MRI contrast reagents, and materials for enhanced transfection cannot be overlooked. Before such products can reach the market, however, the field must not only address the cost of manufacture and quality control of 
pharmaceutical-grade materials, but also assess the longterm human and environmental health consequences of dendrimer exposure in vivo. This review will highlight the structures, synthesis, properties of dendrimers as well as recent studies on the applications of dendrimers.

\section{SYNTHESIS OF DENDRIMERS}

Dendrimers are generally prepared using either divergent method or convergent method. There is a fundamental difference between these two construction concepts. In the divergent methods, dendrimer grows outwards from a multifunctional core molecule. The core molecule reacts with monomer molecules containing one reactive and two dormant groups giving the first generation dendrimer. Then the new periphery of the molecule is activated for reactions with more monomers. The process is repeated for several generations and a dendrimer is built layer after layer (Fig. 1).
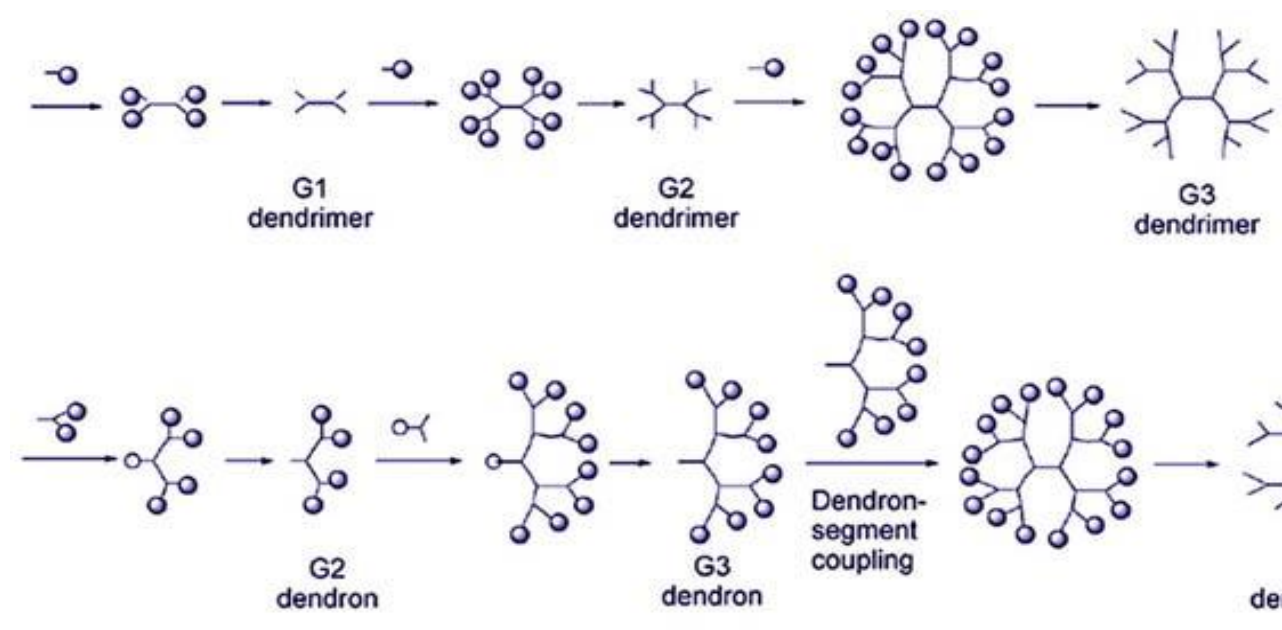

G3

dendron
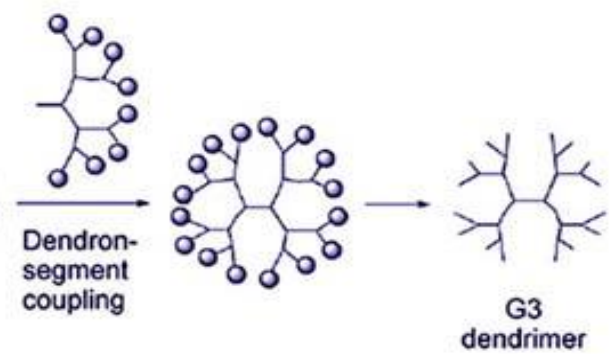

$0=$ Protective group

$\mathrm{O}=$ Orthogonal protective group

Figure 1: Two different strategies of dendrimer synthesis. Top: divergent strategy, bottom: convergent strategy.

The divergent approach is successful for the production of large quantities of dendrimers. Problems occur from side reactions and incomplete reactions of the end groups that lead to structure defects. To prevent side reactions and to force reactions to completion large excess of reagents is required. It causes some difficulties in the purification of the final product.

The convergent methods were developed as a response to the weaknesses of the divergent synthesis. In the convergent approach, the dendrimer is constructed stepwise, starting from the end groups and progressing inwards. When the growing branched polymeric arms, called dendrons, are large enough, they are attached to a multifunctional core molecule (Fig. 1). The convergent growth method has several advantages. It is relatively easy to purify the desired product and the occurrence of defects in the final structure is minimised. It becomes possible to introduce subtle engineering into the dendritic structure by precise placement of functional groups at the periphery of the macromolecule. The convergent approach does not allow the formation of high generations because steric problems occur in the reactions of the dendrons and the core molecule ${ }^{7}$.

The first synthesised dendrimers were polyamidoamines (PAMAMs). They are also known as "starburst" dendrimers (trademark of the Dow Chemicals Company). Ammonia is used as the core molecule which reacts with methyl acrylate in the presence of methanol and then ethylenediamine is added:

$\mathrm{NH}_{3}+3 \mathrm{CH}_{2} \mathrm{CHCOOCH}_{3} \rightarrow \mathrm{N}-\left(\mathrm{CH}_{2} \mathrm{CH}_{2} \mathrm{COOCH}_{3}\right)_{3}$.

$\mathrm{N}-\left(\mathrm{CH}_{2} \mathrm{CH}_{2} \mathrm{COOCH}_{3}\right)_{3}+3 \mathrm{NH}_{2} \mathrm{CH}_{2} \mathrm{CH}_{2} \mathrm{NH}_{2} \rightarrow \mathrm{N}-$

$\left(\mathrm{CH}_{2} \mathrm{CH}_{2} \mathrm{CONHCH}_{2} \mathrm{CH}_{2} \mathrm{NH}_{2}\right)_{3}+3 \mathrm{CH}_{3} \mathrm{OH}$
The free amino group present at the end react with two methyl acrylate monomers and two ethylenediamine molecules producing new dendrimer generation. The halfgeneration PAMAM dendrimers (e.g., 0.5, 1.5, and 2.5) possess anionic surfaces of carboxylate groups. The number of reactive surface sites is doubled with every generation and the mass increases more than twice. Dendritech TM (U.S.A.) manufactures PAMAM dendrimers. These are based on either an ethylenediamine (EDA) core or ammonia core and possess amino groups on the surface. They are usually sold as a solution in either methanol or water. DSM (Netherlands) has developed production of poly (propylene imine) dendrimers. They are currently available under the name Astramol TM. Butylenediamine (BDA) is used as the core molecule. The repetitive reaction sequence involves Michael addition of acrylonitrile to a primary amino group followed by hydrogenation of nitrile groups to primary amino groups 8 .

\section{MOLECULAR STRUCTURE OF DENDRIMER}

Dendrimers have a globular configuration with monomer units branching out from a centre core. The structure is highly defined and organized. The number of branches increases exponentially extending from core to the periphery. The branching would come to a stop when the steric hindrance would come to a stop any further growth. In general, dendrimers consists of three major regions (a) an initiator core, (b) a shell with extending arms or branches made of building blocks and (c) the exterior or outer-most surface groups on the termini of the branches 9 . The monomers that attach to the core form the first branches (also called First Generation). The three parts of the dendrimer can be specifically tailored towards a 
desired molecular property or function of the dendrimer such as drug delivery, molecular sensors, enzyme mimics, etc.

Dendrimers of lower generations (0, 1, and 2) have highly asymmetric shape and possess more open structures as compared to higher generation dendrimers. As the chains growing from the core molecule become longer and more branched (in 4 and higher generations) dendrimers adopt a globular structure as represented in Figure 2 . Dendrimers become densely packed as they extend out to the periphery, which forms a closed membrane-like structure. When a critical branched state is reached dendrimers cannot grow because of a lack of space. This is called the 'starburst effect'. For PAMAM dendrimer synthesis it is observed after tenth generation. The rate of reaction drops suddenly and further reactions of the end groups cannot occur. The tenth generation PAMAM contains 6141 monomer units and has a diameter of about $124 \AA$. The increasing branch density with generation is also believed to have striking effects on the structure of dendrimers. They are characterised by the presence of internal cavities and by a large number of reactive end groups.

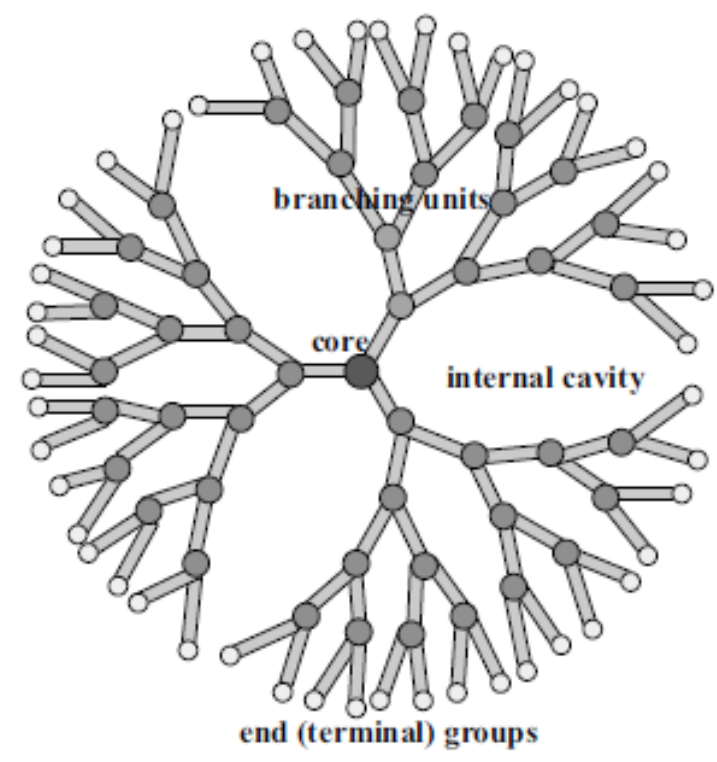

Figure 2: Representation of a Fourth Generation Dendrimer.

\section{PHYSICOCHEMICAL PROPERTIES OF DENDRIMERS}

\section{- Monodispersity and Rheological Properties}

Dendrimers are monodisperse macromolecules, unlike linear polymers synthesized step-by-step, have wellorganized structures with a very low polydispersity index $(\mathrm{Mw} / \mathrm{Mn}<1.01-1.05)$. The classical polymerization process which results in linear polymers is usually random in nature and produces molecules of different sizes, whereas size and molecular mass of dendrimers can be specifically controlled during synthesis ${ }^{10}$. Because of their molecular architecture, dendrimers show some significantly improved physical and chemical properties when compared to traditional linear polymers 11. In solution, linear chains exist as flexible coils; in contrast, dendrimers form a tightly packed ball. This has a great impact on their rheological properties. Dendrimer solutions have significantly lower viscosity than linear polymers. When the molecular mass of dendrimers increases, their intrinsic viscosity goes through a maximum at the fourth generation and then begins to decline. Such behaviour is unlike that of linear polymers.

\section{- Solubility and Reactivity}

The solubility of dendrimers is determined by the surface functional groups, dendrimer generation, repeated units, and even the core. Dendrimers were reported to possess perfect solubility in a large number of solvents. Their high solubility in organic solvents leads to rapid dissolution and provides various approaches to characterize their structures. Also, high water-solubility ensures their applications as solubility enhancers for hydrophobic guest molecules ${ }^{12}$. On the other hand, high density of surface functional groups $(-\mathrm{NH} 2,-\mathrm{COOH},-\mathrm{OH})$ in PAMAM dendrimers may be expected to conjugate with a series of bioactive molecules. These surface-modified dendrimers with different functions provide us with new nanodevice design strategies. Overall, high solubility and reactivity make dendrimers suitable as a platform in biomedical fields 13 .

\section{EFFECT OF VARIOUS FACTORS ON PROPERTIES OF DENDRIMERS}

- $\quad$ Effect of pH: Amino-terminated PPI and PAMAM dendrimers have basic surface groups as well as a basic interior. For these types of dendrimers with interiors containing tertiary amines, the low $\mathrm{pH}$ region generally leads to extended conformations due to electrostatic repulsion between the positively charged ammonium groups. Applying molecular dynamics to predict the structural behaviour of PAMAM dendrimers as a function of $\mathrm{pH}$ show that the dendrimer has an extended conformation, based on a highly ordered structure at low $\mathrm{pH}(\mathrm{pH} \leq 4)$. At this $\mathrm{pH}$, the interior is getting increasingly "hollow" as the generation number increases as a result of repulsion between the positively charged amines both at the dendrimer surface and the tertiary amines in the interior. At neutral pH, back-folding occurs which may be a consequence of hydrogen bonding between the uncharged tertiary amines in the interior and the positively charged surface amines. At higher $\mathrm{pH}(\mathrm{pH} \geq 10)$ the dendrimer contract as the charge of the molecule becomes neutral, acquiring a more spherical (globular) structure based on a loose compact network, where the repulsive forces between the dendrimer arms and between the surface groups reaches a minimum ${ }^{14}$. At this $\mathrm{pH}$, the conformation has a higher degree of back-folding as a consequence of the weak "inter-dendron" repulsive forces (Figure 3). Dendrimers (G5-G7) are conformationally more affected by change in $\mathrm{pH}$ and ionic strength in comparison to higher generation dendrimers (e.g. G8). The reason for this may be found in the somewhat more restricted motion of the outer shell chain segments in the higher generation dendrimers, leading to a more globular-shaped molecule despite different conditions in the surroundings 15 . 

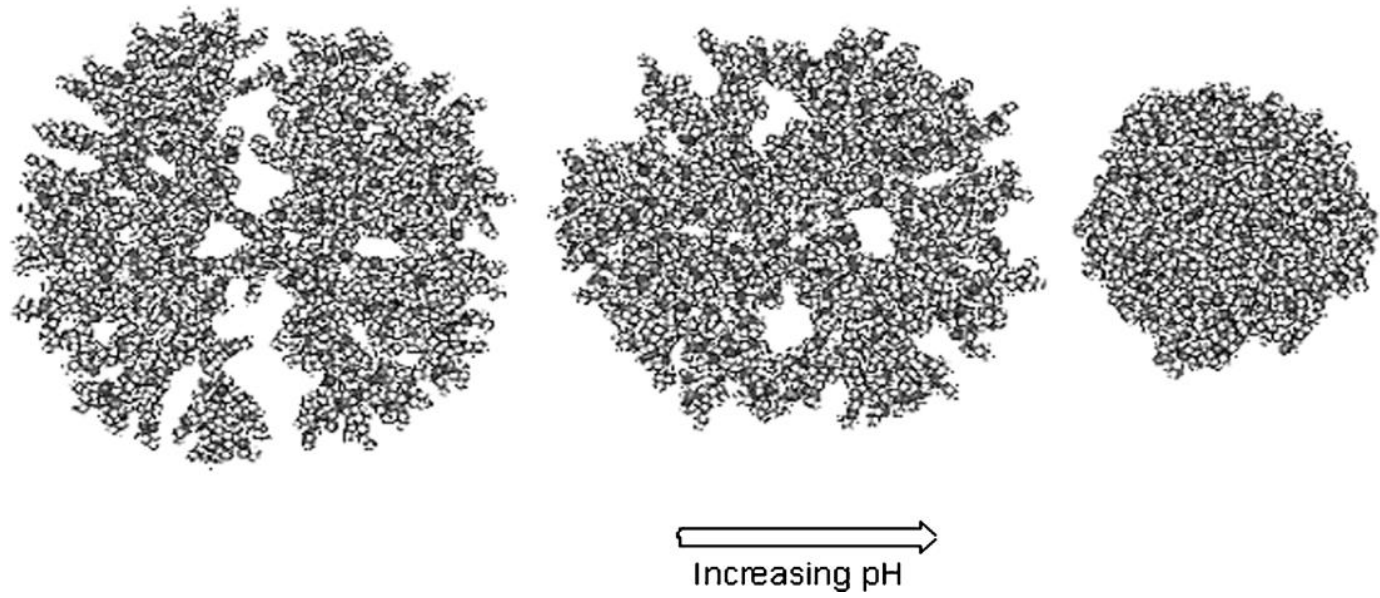

Figure 3: Three-dimensional structure of a G6-PAMAM dendrimer, under different pH.

- $\quad$ Effect of solvent: The ability of the solvent to solvate the dendrimer structure is a very important parameter when investigating the conformational state of a dendrimer. Molecular dynamics has been applied to study the variation of dendrimer conformation as a function of dendrimer generation in different solvents. Dendrimers of all generations generally all experience a larger extend of back-folding with decreasing solvent quality, i.e. decreasing solvation. However, being more flexible, the low generation dendrimers show the highest tendency towards backfolding as a result of poor solvation compared to the higher generation dendrimers. NMR studies performed on PPI dendrimers conclude that an apolar solvent like benzene, poorly solvates the dendrons favouring intramolecular interactions between the dendrimer segments and backfolding. However, a weakly acidic solvent like chloroform can act as a hydrogen donor for the interior amines in a basic dendrimer like PPI, leading to an extended conformation of the dendrimer because of extensive hydrogen bonding between the solvent and the dendrimer amines 16 . Both experimental as well as theoretical studies on amino-terminated PPI and PAMAM dendrimers (polar dendrimers) show the tendency that apolar aprotic ("poor") solvents induce higher molecular densities in the core region as a result of back-folding, whereas polar ("good") solvents solvate the dendrimer arms and induce a higher molecular density on the dendrimer surface. Interestingly, dendrimers having polar surface groups to some extent resemble proteins in their conformational behaviour when subjecting these structures to more apolar conditions, in the sense that back-folding of the polar surface groups may expose the more hydrophobic dendrimer parts to the surroundings leading to a decreased surface polarity of the back-folded dendrimer. A similar behaviour has been observed in the adsorption of proteins onto hydrophobic surfaces ${ }^{17}$, giving a highly denaturated (unfolded) state of the protein exposing its interior hydrophobic regions to interact with the surface (Figure 4).

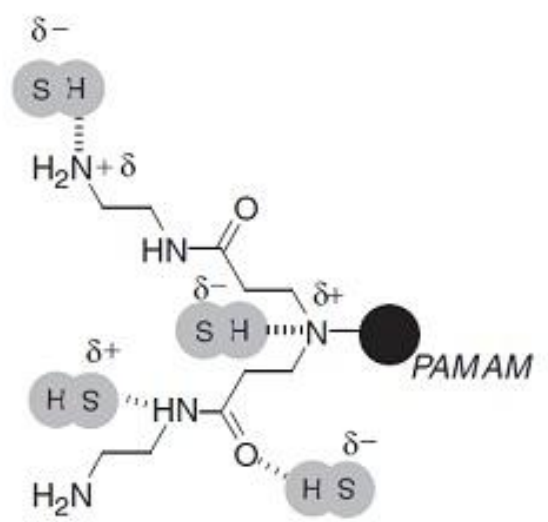

H S $=$ Protic solvent

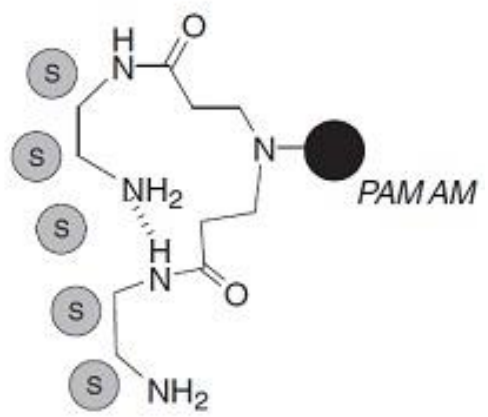

(s) = Apolar solvent

Figure 4: Proposed scheme for solvation of a dendrimer under different solvent conditions.

Left: Solvation of a polar dendrimer in a protic solvent ("good"), solvent leading to extended conformation exposing a polar surface.

Right: Polar dendrimer in an apolar aprotic solvent ("poor"), solvent leading to exposure of an apolar surface consisting of alkyl chains by back-folding. 
- Effect of Salt: Molecular simulations generally conclude that high ionic strength (high concentration of salts) has a strong effect on charged PPI dendrimers and favours a contracted conformation of dendrimers, with a high degree of back-folding somewhat similar to what is observed upon increasing $\mathrm{pH}$ or poor salvation 18. At low salt conditions, the repulsive forces between the charged dendrimer segments results in an extended conformation in order to minimise charge repulsion in the structure (Figure 5).

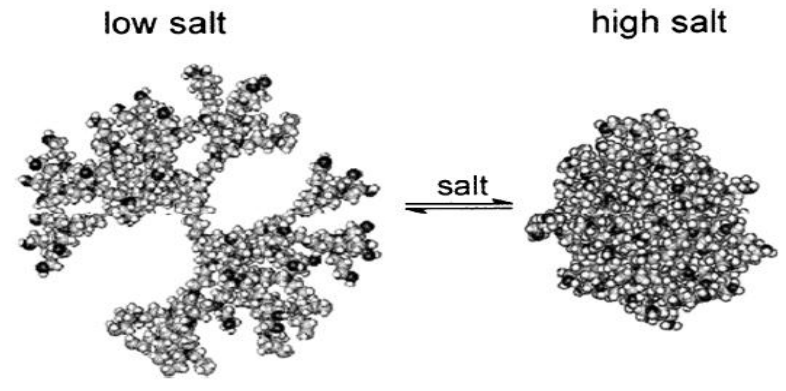

Figure 5: Showing the three-dimensional conformational change of a PPI dendrimer upon increasing ionic strength

- $\quad$ Effect of Concentration: In dendrimers with flexible structures the conformation is not only affected by small molecules like solvents, salts or protons, but may also be sensitive to larger objects, such as other dendrimers or surfaces which can have a great affect on the molecular density and conformation of the dendrimer. Small angle Xray scattering (SAXS) experiments performed on PPI dendrimers $(G 4, G 5)$ in a polar solvent like methanol show that the molecular conformation of dendrimers upon increasing concentration becomes increasingly contracted. This molecular contraction may minimise the repulsive forces between the dendrimer molecules and increase the ability of the dendrimers to exhibit a more tight intermolecular packing ${ }^{19}$.

\section{CHARACTERIZATION OF DENDRIMERS}

The characterization of dendrimers is rather complex due to the size and symmetry of these macromolecules. Various NMR techniques $(1 \mathrm{H}, 13 \mathrm{C}, 15 \mathrm{~N}, 31 \mathrm{P})$, elemental analyses, and chromatography techniques (HPLC, SEC) are widely used, but these techniques cannot reveal small amounts of impurities in, especially, higher generation dendrimers. Fortunately, recent progress in ESI (electrospray ionization) and MALDI (matrix-assisted laser desorption ionization) mass spectrometry allows for an in-depth analysis of dendrimers. ESI-MS has been used to identify the imperfections in both poly(propylene imine) and poly(amidoamine) dendrimers (PAMAM). Both of these dendrimer types are made via a divergent synthesis and are very suitable for electro spray ionization due to their polar and basic nature 20 .

\section{- Spectroscopy Techniques}

Nuclear Magnetic Resonance (NMR): NMR is mainly used for analyzing step by step synthesis of dendrimer, to probe the size, morphology, dynamics of dendrimers for organic dendrimers such as PPI and polyphenylester.

UV-Visible Spectroscopy: UV-Visible Spectroscopy can be used to monitor the synthesis of dendrimers, The intensity of the absorption band is essentially proportional to the number of chromophoric units, and can be a test for the purity of PPI dendrimers having azobenzene as end groups, for phosphorus dendrimers having azobenzenes within the branches, or double-layered carbosilane dendrimers 21,22 .

Infra-red (IR) and Raman Spectroscopy: Infra-red spectroscopy is mainly used for routine analysis of the chemical transformations occurring at the surface of dendrimers, such as the disappearance of nitrile groups in the synthesis of PPI dendrimers, the occurrence of hydrogen bonding in PPI glycine functionalized dendrimers or the disappearance of the aldehydes during synthesis of $\mathrm{PMMH}$ dendrimers. Infra red spectroscopy (IR) for routine analysis of the chemical transformations occurring at the surface of dendrimers and to characterize delocalize $\pi-\pi$ stacking interaction between end groups of modified PAMAM. Raman spectroscopy gave relevant information about the degree of cyclodehydrogenation of polyphenylene dendrimers, and the characterization of PPI and phosphorus dendrimers.

Fluorescence: The high sensitivity of fluorescence has been used to quantify defects during the synthesis of dendrimers, such as unreacted $\mathrm{CO}_{2} \mathrm{H}$ groups in $\mathrm{ARB}$ dendrimers, but its main use is to characterize the structure of dendrimers having photochemical probes covalently linked to one particular section.

$X$-ray diffraction: This technique should allow precise determination of the chemical composition, size and shape of dendrimers.

Mass spectrometry: Classical Mass Spectrometry techniques such as chemical ionization or fast atom bombardment (FAB) can be used only for the characterization of small dendrimers, whose mass is below 3000 D.

\section{- Scattering Techniques}

Small angle X-ray scattering (SAXS) gives information about their average radius of gyration (Rg) in solution. The intensity of the scattering as a function of angle also provides information on the arrangement of polymer segments, hence on the segment density distribution within the molecule 23 .

Small angle neutron scattering (SANS) gives access to the radius of gyration, but may also reveal more accurate information than SAXS about the internal structure of the entire dendrimer. The location of the end groups has also been determined by SANS experiments conducted with PAMAM dendrimers and PPI dendrimers having labelled (deuterated) or unlabelled end groups.

Laser light scattering (LLS) to determine the hydrodynamic radius of dendrimers, Dynamic LLS is used for the detection of aggregates.

\section{- Microscopy Methods}

Transmission Electron Microscopy and Scanning Electron Microscopy are mainly used for the imaging of dendrimers. Visualizing single molecules by optical microscopy has been successfully carried out for dendrimers having a fluorescent core. Confocal microscopy allowed one to observe the fluorescence of a third generation PBzE dendrimer having a dihydropyrrolo pyrroledione as a core and polyphenylene dendrimers having peryleneimide as end groups. 


\section{- Size Exclusion Chromatography}

Size Exclusion Chromatography (SEC) allows the separation of molecules according to size. A detector such as a differential refractive index or a LLS detector is connected to the SEC apparatus for the determination of the polydispersity, which is generally very close to unity. Most types of dendrimers are characterized by SEC, even self assembled dendrimers 24

\section{- Electrical Techniques}

Electron paramagnetic resonance (EPR) is the Quantitative determination of substitution efficiency on the surface of PAMAM dendrimers 25.

Electrochemistry gives information about the possibility of interaction of electroactive end groups.

Electrophoresis used for the assessment of puritity and homogeneity of several types of water soluble dendrimers.

Electro Spray Ionisation (ESI) can be used for dendrimers able to form stable multicharged species. It has been applied to PPI dendrimers and to PAMAM dendrimers up to generations. Electrospray ionization can be used for dendrimers able to form stable multicharged species 26 .

\section{- Rheology And Physical Properties}

Intrinsic viscosity: Rheology, and particularly dilute solution viscometric studies, can be used as analytical probe of the morphological structure of dendrimers.

Differential Scanning Calorimetry (DSC): The DSC technique is generally used to detect the glass transition temperature (Tg), which depends on the molecular weight, entanglement and chain-end composition of polymers. The $\mathrm{Tg}$ is affected by the end group substitutions, and the molecular mass of dendrimers.

Dielectric Spectroscopy (DS): Dielectric Spectroscopy gives information about molecular dynamic processes in polymers (a-, h-, g- and y-relaxation) ${ }^{27}$.

\section{- Miscellaneous}

X-ray Photoelectron Spectroscopy (XPS): chemical composition of dendrimers such as poly (aryl ether) dendrons or PMMH dendrimers has been also obtained using XPS, this technique is most generally used for the characterization of layers, Sedimentation for lactosylated PAMAM dendrimers, measurements of dipole moments for PMMH dendrimer. Titrimetry used to determine the number of $\mathrm{NH}_{2}$ end groups of PAMAM dendrimers.

\section{APPLICATIONS OF DENDRIMERS}

\section{- Pharmaceutical Application}

Dendrimers in Oral Drug Delivery: Dendrimers may act as potential candidates for orally controlled release systems by conjugating/encapsulating drug molecules to/in them. They allow the maintenance of drug concentrations within the therapeutic range at the injured regions, and hence can simplify dosing schedules. In addition, dendrimers can significantly increase the solubility of orally administrated drugs and even the stability of drugs in biological environments. These macromolecules with bioadhesive properties have strong affinity for mucosa and can prolong the residence time of the orally administrated drug in contact with the intestinal epithelium. Furthermore, dendrimers themselves can easily penetrate through intestinal membranes, and thus can enhance the oral absorption of low-penetration drugs. These properties make dendrimers suitable carriers for the development of oral drug delivery systems ${ }^{28}$. It was suggested that colloidal drug carriers such as dendrimers could be absorbed by way of the Peyer's patches, which is a route to enhance the oral absorption of encapsulated drug molecules and to minimize enzymatic degradation in the intestine tissue ${ }^{29}$. Experimental results showed a preferential uptake of dendrimers through the lymphoid tissue in the small intestine but not in the large intestine. The percentage of the initial dose absorbed through the lymphoid tissue was comparatively greater than that through the nonlymphoid tissue of the small intestine. These results indicated that dendrimers were capable to enhance the absorption of low penetration drugs in the small intestine tissues. Dendrimer size was a key factor on determining overall uptake. Macromolecules with diameters up to $3 \mathrm{~nm}$ may penetrate through the intestinal membranes via either the transcellular or paracellular pathway ${ }^{30}$. Therefore, G2.5 and G3.5 PAMAM dendrimers could transport across the intestine via these ways. On the other hand, G4 and higher generation PAMAM dendrimers could attach to the invaginating plasma membrane, and enter cells by specific or nonspecific adsorptive endocytosis. G2.5 and G3.5 PAMAM dendrimers showed particularly low tissue uptake ability, while G5.5 PAMAM dendrimer displayed a higher tissue accumulation than G2.5 and G3.5 dendrimers. Moreover, cationic dendrimers showed a different pattern of accumulation from anionic dendrimers. The negatively charged cell membrane could interact strongly with cationic molecules, and hence led to higher tissue association and lower transport rate of these dendrimers. These results indicated that dendrimers exhibited a size/conformation/charge sensitivity of the transport mechanism across the intestine 31 .

5-FU, a well-known anti-cancer drug, has high toxic side effects in clinical practice. Several attempts have been done to reduce its toxicity by using polymeric carriers. Tripathi et al. 32 modified the surface groups of PAMAM dendrimers with fatty acid molecules and then coated the obtained dendrimer grafts by phospholipids. The dendritic structures were then used to encapsulate 5-FU and employed as potential oral drug carriers. In vivo pharmacokinetic parameters and bioavailability were determined from the blood concentration of 5-FU performed in albino rats. The dendrimer-drug formulation was found to be significantly more effective than free drugs after oral administration.

Dendrimers in Transdermal Drug Delivery: PAMAM dendrimers can improve either the water-solubility or stability of hydrophobic drugs. These materials with hydrophilic outer shells and hydrophobic interiors, which accord with structural requirement of polymeric transdermal enhancers, are expected to act as effective penetration enhancers. Wang et al. 33 reported the utilization of polyhydroxyalkanoate (PHA) and G3 PAMAM dendrimer as a novel TDDS. PHA used by researchers was composed of 3-hydroxyhexanoic acid (8\%) and 3hydroxyoctanoic acid (92\%). Before the in vitro permeation experiments was performed on snake skins, PHA and the model drug (tamsulosin hydrochloride) were mixed together and laid on the transdermal delivery patches. In order to determine the effect of the PAMAM dendrimer on in vitro penetration efficiency of tamsulosin hydrochloride, they pretreated the snake skin with PAMAM dendrimer solution for $24 \mathrm{~h}$. However, no significant enhancement of permeation amount of the 
model drug was found. When PAMAM dendrimer was coadministrated with the PHA matrix, the penetration amount of tamsulosin in the dendrimer-containing PHA matrix was $24.0 \mathrm{mg} / \mathrm{cm}^{2} /$ day while that in the dendrimerlacking PHA matrix was $15.7 \mathrm{mg} / \mathrm{cm}^{2} /$ day (the required amount of this drug in clinical trials is $20 \mathrm{mg} / \mathrm{cm} 2 /$ day). Thus, the authors concluded that PHA-dendrimer matrix reached the clinical aim and could be developed as a useful delivery system for clinical TDDS. In a separate experiment, the same team reported the mechanism of enhancement effect of dendrimer on drug penetration through the skin in TDDS ${ }^{34}$. G3 PAMAM dendrimer with amino groups and G2.5 PAMAM dendrimer with carboxyl groups were used. The results showed that only cationic dendrimers could increase the penetration ability of tamsulosin hydrochloride through the skin. To clarify the mechanism of this enhancement effect by cationic dendrimers, the authors accidentally found that tamsulosin hydrochloride formed crystal in the cationic dendrimer/drug formulation by X-ray diffraction and hence suggested that the crystallization reduced the drug diffusion direction and improved a highly ordered orientation.

Cheng YY et al. 35 investigated transdermal delivery of nonsteroidal anti-inflammatory drugs mediated by PAMAM dendrimers. Ketoprofen and Diflunisal were used as model drugs. In vitro permeation studies on excised rat skins indicated that PAMAM dendrimers significantly enhanced the steady-state flux of both drugs, compared to the drug suspensions without PAMAM dendrimers. A possible explanation for this transdermal-enhancing effect is that dendrimers increased the water solubility of NSAIDs therefore solubilities of both ketoprofen and diflunisal was dramatically increased in the presence of PAMAM dendrimers. Alternatively, the large number of primary amines with cationic charges on the surface of dendrimers could conceivably alter the skin barrier function and enable NSAIDs-PAMAM dendrimer complexes to pass through.

Dendrimers in Ocular Drug Delivery: The main challenge in ocular drug delivery is to increase the drug bioavailability and prolong the residence time of the drug on the cornea, conjunctiva, and corneal epithelia. Up to now, different polymeric formulations, such as natural polymers, bioadhesive polymers, and colloidal formulations, have been used as potential ophthalmic drug carriers 36. These polymeric formulations with perfect viscosity can prolong the drugs' residence time on the cornea and increase their bioavailability. However, most of these formulations give rise to unwanted side effects 37 . Polymeric nanoparticles tend to be removed by lachrymal drainage whereas microparticulates of larger sizes are less tolerable because they may cause eye irritation and tend to be eliminated by the flow of tears. Additionally, administration of these polymers to the cornea may lead to blurred vision resulting from infiltration of the lachrymal gland with round cells and reduced secretion of lachrymal fluid. Overall, development of new functional materials to avoid these problems still holds the key for the future ocular drug delivery. Recently, dendrimers with distinct properties from traditional polymers were suggested to act as ophthalmic vehicles in ocular delivery systems. These dendritic polymers might dissolve hydrophobic drugs in their cavities and accomplish both retention and sustained/controlled release of drugs. Vandamme and Brobeck 38 have reported the development of using PAMAM dendrimers as ophthalmic vehicles in ocular delivery systems. Pilocarpine nitrate and Tropicamide were employed as model drugs for miotic and mydriatic activity tests, respectively. Under the same experimental conditions, the mean ocular residence times of PAMAM dendrimer aqueous solutions (G1.5, G2- OH, G4-OH)) were found to be comparable to that of the $0.2 \% \mathrm{w} / \mathrm{v}$ Carbopo 1 solution, which is a well-known bioadhesive polymer for ophthalmic dosage forms. The ocular residence time of the G2 dendrimer solution was even significantly longer than that of Carbopol or HPMC solutions. The results obtained from the miotic and mydriatic activity tests on rabbits indicated that the pharmacological activities of pilocarpine nitrate and tropicamide were much greater when they were co-administrated with PAMAM dendrimers. The eye drops containing PAMAM dendrimers were also found to have a prolongation of miotic/mydriatic activity. All the three PAMAM dendrimer solutions (Gn-NH2, Gn-OH, and $\mathrm{Gn}-\mathrm{COOH}$ ) significantly improved the bioavailability of pilocarpine nitrate and tropicamide when compared with phosphate solutions containing these drugs. The authors explained that the increased bioavailability of pilocarpine nitrate was neither contributed to the difference of $\mathrm{pH}$ conditions in the reference solutions nor associated with the interactions between the dendrimers and drug molecules. However, it might be contributed to (1) the host-guest relationship between dendrimers and drug molecules, which induced slower release of these drugs encapsulated in dendrimers' interior cavities and (2) the bioadhesive properties of PAMAM dendrimers, which can be explained by the structure, shape, and surface functional groups of dendrimers. The influence of dendrimer concentration and surface functional groups for controlled ocular drug delivery was also determined, and the results suggested that dendrimer size, molecular weight, charge intensity, and molecular geometry influenced ocular delivery efficiency.

Shaunak et al. [39] used carboxylated PAMAM dendrimer (G3.5) to synthesize dendrimer-glucosamine (DG) and dendrimer-glucosamine 6-sulfate (DGS) conjugates with immuno-modulatory and anti-angiogenic properties, respectively. These two conjugates were used together in a validated and clinically relevant rabbit model of wound healing after glaucoma filtration surgery to increase the long-term success of the surgery and prevent scar tissue formation. DG $(60.30 \mathrm{mg} /$ animal) and DGS (30.15 $\mathrm{mg} /$ animal) were administered by subconjunctival injection. Bleb survival was evaluated as the primary efficacy end point of treatment. The results showed that combination subconjunctival treatment with DG and DGS increased the long-term success of the surgery from 30\% to $80 \%$ and inhibited the pro inflammatory and proangiogenic responses in these animals. Rabbits treated with DG and DGS showed minimal scar tissue formation compared to placebo-treated animals. Furthermore, no clinical, haematological, or biochemical toxicity, or microbial infections were found in all animals during the whole experimental period. The results suggested that ocular administration of DG and DGS might be effective, reasonable, and safe routes in clinical practice.

As estimated by the World Health Organization (WHO), corneal diseases are the major causes of blindness. Although traditional allograft cornea transplantation has a satisfactory success rate, lack of corneal tissue is still the major problem which prevents these blind people from the light. Therefore, it is desirable to find artificial corneas that can be able to replace the damaged ones in patients. Recently, Duan and Sheardown ${ }^{40}$ used G2 PPI dendrimer 
to prepare highly cross-linked collagen with mechanical properties and applied it as a corneal tissue-engineering scaffold. The artificial biomaterial was shown to have much better mechanical properties, adhesion ability, optical transparency, and glucose permeability than natural human cornea as well as EDC and glutaraldehyde crosslinked collagen. In vitro culture of human corneal epithelial cells on dendrimer cross-linked collagen gels was used to confirm that the presence of dendrimers did not adversely affect the biocompatibility of the gels. The results suggested dendrimer-cross-linked collagen supported the human corneal epithelial cell growth and adhesion without cell toxicity. Overall, these results indicated that dendrimers could be successfully applied for collagen crosslinking to produce transparent, mechanically stronger, and more biocompatible collagen gels. Dendrimer cross-linked collagen gels can also be used to develop various collagen-based tissue engineering scaffolds and may be applied as suitable scaffolds for artificial corneal and many other tissue engineering applications.

Dendrimers in Targeted Drug Delivery: Star-burst dendrimers represent a superior carrier platform for targeted drug delivery. Yang et al. ${ }^{41}$ reported targeting cancer cells with biotin-dendrimer conjugates. Partially acetylated generation 5 (G5) polyamidoamine (PAMAM) dendrimer was conjugated with the targeting moiety (biotin) and the imaging moiety (fluoresceinisothiocyanate, FITC), and the flow cytometry and confocal microscopy study revealed that resulting dendrimer-biotin -FITC conjugate exhibited much higher cellular uptake into HeLa cells than the conjugate without biotin. The uptake was energy-dependent, dose-dependent, and could be effectively blocked by dendrimer-conjugated biotin. Therefore biocompatible biotin-dendrimer conjugate might be a promising nano-platform for cancer therapy and cancer diagnosis.

Dendrimers can also be used as coating agents to protect or deliver drugs to specific sites in the body or as timerelease vehicles for biologically active agents. 5Fluorouracil (5FU) is known to have remarkable antitumour activity, but it has high toxic side effects. PAMAM dendrimers after acetylation can form dendrimer5FU conjugates. The dendrimers are water soluble and hydrolysis of the conjugates releases free 5FU. The slow release reduces $5 \mathrm{FU}$ toxicity. Such dendrimers seem to be potentially useful carriers for antitumour drugs 42 .

Choi et al. 43 reported Dendrimer-based multivalent vancomycin nanoplatform for targeting the drug-resistant bacteria surface. Vancomycin represents the preferred ligand for bacteria-targeting nanosystems. However, it is inefficient for emerging vancomycin-resistant species because of its poor affinity to the reprogrammed cell wall structure. Therefore resarchers reported the use of a multivalent strategy as an effective way for overcoming such an affinity limitation in bacteria targeting. They designed a series of fifth generation (G5) poly(amidoamine) (PAMAM) dendrimers tethered with vancomycin at the $\mathrm{C}$-terminus at different valencies and performed surface plasmon resonance (SPR) studies to determine their binding avidity to two cell wall models, each made with either a vancomycin-susceptible (D)-Ala(D)-Ala or vancomycin-resistant (D)-Ala-(D)-Lac cell wall precursor. These conjugates showed remarkable enhancement in avidity in the cell wall models tested, including the vancomycin-resistant model, which had an increase in avidity of four to five orders of magnitude ISSN: 2250-1177 greater than free vancomycin. The tight adsorption of the conjugate to the model surface corresponded with its ability to bind vancomycin-susceptible Staphylococcus aureus bacterial cells in vitro were imaged by confocal fluorescent microscopy. This vancomycin platform was then used to fabricate the surface of iron oxide nanoparticles by coating them with the dendrimer conjugates, and the resulting dendrimer-covered magnetic nanoparticles were demonstrated to rapidly sequester bacterial cells. Thus the researchers successfully investigates the biophysical basis of the tight, multivalent association of dendrimer-based vancomycin conjugates to the bacterial cell wall, and proposed a potential new use of this nanoplatform in targeting Gram-positive bacteria.

Dendrimers for Controllable Gene Therapy/Non-Viral Gene Delivery: Dendrimers can act as carriers, called vectors, in gene therapy. Vectors transfer genes through the cell membrane into the nucleus. Currently liposomes and genetically engineered viruses have been mainly used for this. PAMAM dendrimers have also been tested as genetic material carriers ${ }^{44}$. They are terminated in amino groups which interact with phosphate groups of nucleic acids. This ensures consistent formation of transfection complexes. A transfection reagent called SuperFect TM consisting of activated dendrimers is commercially available. Activated dendrimers can carry a larger amount of genetic material than viruses. SuperFect-DNA complexes are characterised by high stability and provide more efficient transport of DNA into the nucleus than liposomes. The high transfection efficiency of dendrimers may not only be due to their well-defined shape but may also be caused by the low pK of the amines (3.9 and 6.9). The low $\mathrm{p} K$ permit the dendrimer to buffer the $\mathrm{pH}$ change in the endosomal compartment 45 . PAMAM dendrimers functionalized with cyclodextrin showed luciferase gene expression about 100 times higher than unfunctionalized PAMAM or non-covalent mixtures of PAMAM and cyclodextrin. Dendrimers of high structural flexibility and partially degraded high-generation dendrimers (i.e., hyper branched architectures) appear to be better suited for certain gene delivery operations than intact highgeneration symmetrical dendrimers 46 .

\section{Dendrimers as Bio Mimetic Artificial Proteins and Vaccines}

Dendrimers are often referred to as "artificial proteins" due to their dimensional length scaling, narrow size distribution, and other bio mimetic properties 47 . For examples PAMAM family, they closely match the sizes and contours of many important proteins and bio assemblies like insulin $(3 \mathrm{~nm})$, cytochrome C ( $4 \mathrm{~nm})$, and haemoglobin $(5.5 \mathrm{~nm})$ are approximately the same size and shape as ammonia-core PAMAM dendrimers generations 3,4 and 5, respectively. Generation 2 dendrimer matches the width $(2.4 \mathrm{~nm})$ of DNA duplexes (form stable complexes with histone clusters to condense and store DNA within the nucleosome of cells.) and generations 5 and 6 PAMAM dendrimers have diameters approximately equivalent to the thickness of lipid bilayer membranes $(\sim 5.5 \mathrm{~nm})$ of biological cells. Unlike the classic polymers, dendrimers are rather monodispersed (polydispersity close to 1.00), which makes them very similar to biomacromolecules such as proteins. They can be synthetically designed to mimic various biological functions of metalloproteins and enzymes such as supramolecular self-assembly, molecular recognition and complexation, etc. ${ }^{48}$. Researchers have designed an amphiphilic peptide dendrimer as a synthetic AIDS Vaccine, capable of forming liposomes or micelles by 
attaching tripalmitoy1s-glycery1 cysteine group to a tetravalent peptide antigen containing glycoprotein gp 120 of HIV-1 virus. Starburst dendrimers used for enhanced performance and flexibility for immunoassays. On the basis of feasibility studies with model systems it was concluded that immunoassays can be developed with performance equivalent to or better than that in many established systems and demonstrated enhanced sensitivity for creatinnie kinase MB isoenzyme (CKBB), thyrotropin and myoglobin assays and reduced instrumental analysis time for the CKMB assay.

\section{Boron Neutron Capture Therapy (BNCT)}

In this method, the patient is first injected with a pharmaceutical which moves to cancer cells. This drug contains a stable isotope of boron, to which dendrimers have been attached with covalent bonds 49 . Then, a neutral beam of low energy/thermal electrons is applied to the patient, which in turn destroys the tumor but leaves the healthy cells behind. Such an innovation is so crucial to cancer treatment because current methods such as chemotherapy and radiation therapy cause much harm to healthy cells instead of just cancerous ones. Boronated starburst PAMAM (i.e. polyamidoamine) dendrimermonoclonal antibody immunoconjugates have been used as potentially efficient anti-cancer reagents involving boron capture of neutron (Figure 6).

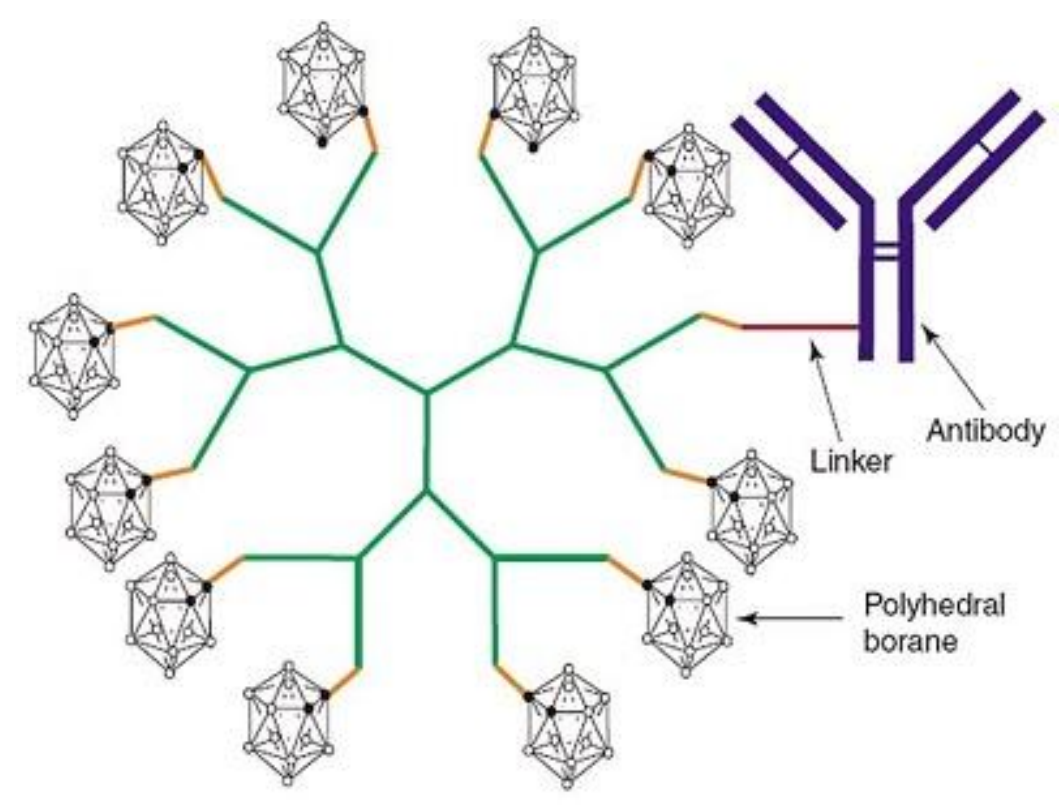

Figure 6: Antibody-Targeted Dendritic Nanodevice for Boron Neutron Capture Therapy

\section{Dendrimer as Solubility Enhancers}

There are many substances which have a strong therapeutic activity but, due to their lack of solubility in pharmaceutically acceptable solvents, they have not been used for therapeutic purposes. Water soluble dendrimers are capable of binding and solubilising small acidic hydrophobic molecules with antifungal or antibacterial properties. Dendrimers having a hydrophobic core and a hydrophilic surface layer, have been termed unimolecular micelles. Unlike traditional micelles, dendrimers do not have a critical micelle concentration. This characteristic offers the opportunity to solubilize poorly soluble drugs by encapsulating them within the dendritic structure at all concentrations of dendrimer. A hydrophilic- hydrophobic core-shell dendrimer with PAMAM interior and long alkane chain exterior was shown to bind 5-flurouracil, a watersoluble anti-tumor drug ${ }^{50}$. After a phospholipid coating of the dendrimer-fatty-acid macromolecule, oral bioavailability in rats of 5-flurouracil was nearly twice the level of free 5-flurouracil. Dendrimer-based carriers could offer the opportunity to enhance the oral bioavailability of problematic drugs. Propranolol when conjugated to surface of modified G3 PAMAM dendrimer, the solubility of propranolol increased by over two orders of magnitude. Thus, dendrimer nanocarriers offer the potential to enhance the bioavailability of drugs that are poorly soluble and/or substrates for efflux transporters.

Exploring Dendrimer towards Dual Drug Delivery with its pH Responsive Simultaneous Drug-Release Kinetics: A major problem associated with conventional leukaemia chemotherapy is the development of resistance that can be surmounted well by combination chemotherapy. Tekade et al. 51 reported a novel technology to load two antileukaemic drugs of choice simultaneously inside the PAMAM dendrimer. Under optimized conditions of $\mathrm{pH}$ and dialysis time, one molecule of PAMAM dendrimer was found to be capable of entrapping $27.02 \pm 0.51$ and $8.00 \pm$ 0.46 molecules of Methotrexate and all-trans Retinoic acid (ATRA), respectively. The simultaneous in-vitro release profiling of the loaded drugs was studied at $\mathrm{pH} \mathrm{4,7.4} \mathrm{and}$ 10. The release kinetics was found to be governed by degree of dendrimer protonation, with more sustained and controlled behaviour at $\mathrm{pH}$ 7.4. Terminal loading of dendrimer with less haemolytic bioactive (ATRA) reduced the haemolytic toxicity of the dendrimer formulation. A cytotoxicity study was also performed on HeLa cell lines by MTT assay, wherein after $72 \mathrm{~h}$, the dual-drug loaded dendrimer was found to be more efficient ( IC $_{50} 0.5 \mu \mathrm{M}$ ) as compared to that of the free drug combination (IC 500.75 $\mu \mathrm{M})$. 


\section{Dendrimer-Based Postnatal Therapy for} Neuroinflammation and Cerebral Palsy in a Rabbit Model: Cerebral palsy (CP) is a chronic childhood disorder with no effective cure. Neuroinflammation, caused by activated microglia and astrocytes, plays a key role in the pathogenesis of $\mathrm{CP}$ and disorders such as Alzheimer's disease and multiple sclerosis. Targeting neuroinflammation can be a potent therapeutic strategy. However, delivering drugs across the blood-brain barrier to the target cells for treating diffuse brain injury is a major challenge. Kannan et al. 52 revealed that systemically administered polyamidoamine dendrimers localized in activated microglia and astrocytes in the brain of newborn rabbits with $\mathrm{CP}$, but not healthy controls. Researchers demonstrated that dendrimer-based N-acetyl-L-cysteine (NAC) therapy for brain injury suppresses neuroinflammation and leads to a marked improvement in motor function in the CP kids. The well-known and safe clinical profile for NAC, when combined with dendrimerbased targeting, provides opportunities for clinical translation in the treatment of neuroinflammatory disorders in humans. The effectiveness of the dendrimerNAC treatment, administered in the postnatal period for a prenatal insult, suggested a window of opportunity for treatment of CP in humans after birth.

\section{Dendrimers in Photodynamic Therapy (PDT)}

Cancer treatment involves the administration of a light activated photosensitizing drug that selectively concentrates in diseased tissue. For example the photosensitizer 5-aminolevulinic acid has been attached to the surface of dendrimers and studied as an agent for PDT of tumorigenic keratinocytes 53 .

- Diagnostic Applications: Dendrimers are the few types of synthetic polymers that can be precisely controlled in size and structure. The well-defined structure renders dendritic macromolecules unique properties for versatile applications, especially in the biomedical field 54 .

Dendrimers as Molecular Probes: Due to their distinct morphology and unique characteristics, dendrimers are used as molecular probes. For Example, the immobilization of sensor units on the surface of dendrimers is a very efficient way to generate an integrated molecular probe, because of their large surface area and high density of surface functionalities 55 .

Dendrimers as $X$-ray contrast agents: Dendrimers are currently under investigation as potential polymeric X-ray contrast agents. Potential dendritic X-ray contrast agents using various organo metallic complexes such as bismuth and tin are used to obtain a high resolution X-ray image, several diseases or organs, such as arteriosclerotic vasculature, tumors, infarcts, kidneys or efferent urinary etc.

Dendrimers as Magnetic Resonance Imaging (MRI) contrast agents: Dendrimer based metal chelates act a magnetic resonance imaging contrast agent. Target specific moieties are attached to the dendritic MRI contrast agents, to improve the pharmacokinetic properties of dendrimer contrast agents, for example folate conjugated Gd (III)DTPA PAMAM dendrimer, which increased the longitudinal relaxation rate of tumor cells expressing the high affinity folate receptor. Dendrimers have the ability to deliver drug inside the cell or they may improve intracellular trafficking. The sixth generation polygadolinium dendrimer displayed a prolonged enhancement time and is extremely useful for 3D time-of-flight MR angiography.
Commercially available dendrimers such as PAMAM and PPI (polypropyleneimine) dendrimer have also been employed as magnetic resonance imaging contrast agents potentially to improve the quality of the clinical diagnostics 56.

Cardiac Testing: Dade International Inc. (U.S.A.) has introduced a new method in cardiac testing. Proteins present in a blood sample bind to immunoglobulins which are fixed by dendrimers to a sheet of glass. The result shows if there is any heart muscle damage. This method significantly reduces the waiting time for the blood test results (to about $8 \mathrm{~min}$ ). When a randomly organised solution of immunoglobulins is used the test lasts up to 40 min. Conjugates of dendrimer and antibody improve also precision and sensitivity of the test.

Biochemical analysis: The design of sugar binding receptor for detection of sugar level in diabetic patients by introducing boronic acid base fluorescence sensor. The anthracene units (chromophore) and boronic acid (sugar bindinig moiety) were attached to second generation PAMAM dendrimer through a tertiary amine. Used for Steric isolation of the methalloporphyrin, this is important to achieve certain biological function 57 .

\section{- Dendritic Catalysts / Enzymes}

Dendrimers are useful as nanoscale catalysts due to its combination of high surface area and high solubility. Dendrimers combine the advantages of homogenous and heterogeneous catalysts. Homogenous catalysts are effective due to a good accessibility of active sites but they are often difficult to separate from the reaction stream. Heterogeneous catalysts are easy to separate from the reaction mixture but the kinetics of the reaction is limited by mass transport. Dendrimers have a multifunctional surface and all catalytic sites are always exposed towards the reaction mixture. They can be recovered from the reaction mixture by easy ultrafiltration methods. Dendritic shells can be used to create a microenvironment which is favourable for catalysis or provide shielding for functional groups at the dendritic core .The first example of a catalytic dendrimer was described by the group of Koten et al. ${ }^{58}$. They terminated soluble polycarbosilane dendrimers in diamino arylnickel (II) complexes. Such dendrimers can be used in addition reactions of polyhaloalkanes.

\section{- Industrial Processes}

Dendrimers can encapsulate insoluble materials, such as metals, and transport them into a solvent within their interior. For example, fluorinated dendrimers, which are soluble in supercritical $\mathrm{CO}_{2}$ and can be used to extract strongly hydrophilic compounds from, water into liquid $\mathrm{CO}_{2}$. This may help develop technologies in which hazardous organic solvents are replaced by liquid $\mathrm{CO}_{2}$.

\section{FUTURE SCOPE OF DENDRIMERS}

The dendrimers holds a promising future in various pharmaceutical applications and diagnostic field in the coming years as they possess unique properties, such as high degree of branching, multivalency, globular architecture and well-defined molecular weight, there by offering scaffold for drug delivery. Specified drug delivery, especially regarding cancer is the primary future application, but there are countless others, including: vectors in gene therapy, solubility enhancers, photodynamic therapy, and industrial processes. Because of their unique cascade architecture, dendrimers can coordinate with metal ions selectively on different 
structural locations such as the surface, core, or branching focal point. These distinct features have attracted great attention among scientists in many disciplines including organic, inorganic, organometallic, polymer, physical and biochemistry as well as materials science. Unique magnetic, electronic and optical properties have been intensively explored in dendritic materials. Dendrimer research has indeed become an interdisciplinary topic with a great influence on wide fields of science and technology. The research endeavours such as a novel organoplatinum antitumor prodrug based on the fourth generation PAMAM dendrimer for potential control-release of active platinum species in cancer treatment by Howell B et al. ${ }^{59}$, Klajnert B et al. 60 investigation on haemotoxicity of PAMAM dendrimers and Chauhan A et al. 61 report on pre-clinical and behavioural toxicity profile of PAMAM dendrimers in mice demonstrate control of molecular properties for a variety of biomedical applications in the design of unique dendrimer structures. Such a task needs the collaborative efforts of synthetic chemists, material engineers and molecular biologists as well as industry experts. Several dendrimer based products have been approved by the FDA and some are in Phase II clinical trials. Various dendrimer based products are:

(1) Alert ticket for Anthrax Detection

\section{REFERENCES}

[1] Aulenta F, Hayes W and Rannard S, Dendrimers: a new class of nanoscopic containers and delivery devices, European Polymer Journal, 2003; (39):1741-71.

[2] Cheng Y, Wang J and Rao T, Pharmaceutical applications of dendrimers: promising nanocarriers for drug delivery, Frontiers in Bioscience, 2008; (13):1447-71.

[3] Boas U and Heegaard PMH, Dendrimers in drug research, Chemical Society Reviews 2004; (33):43-63.

[4] Patri A, Myc A and Beals J, Synthesis and in vitro testing of J591 antibody-dendrimer conjugates for targeted prostate cancer therapy, Bioconjugate Chemistry 2004; (15):1174-78.

[5] Zhu J and Shi X, Dendrimer based nanodevices for targeted drug delivery applications, Journal of Materials Chemistry, 2013 (1):4199-211.

[6] McCarthy TD, Karellas P and Henderson SA, Dendrimers as drugs: discovery and preclinical and clinical development of dendrimer-based microbicides for HIV and STI prevention, Molecular Pharmaceutics, 2005; (2):312-18.

[7] Svenson S: Dendrimers as versatile platform in drug delivery applications. European Journal of Pharmaceutics and Biopharmaceutics 2009; 71:445-62.

[8] Shakti KS, Lohiya GK and Limburkar PP: Dendrimer a versatile polymer in drug delivery. Asian Journal of Pharmaceutics 2009; 3:178-82.

[9] Aulenta F, Hayes W and Rannard S: Dendrimers: A new class of nanoscopic containers and delivery devices. European Polymer Journal 2003; 39:1741-71.

[10] Tomalia DA: Birth of a new macromolecular architecture: dendrimers as quantized building blocks for nanoscale synthetic polymer chemistry. Progress in Polymer Science 2005; 30:294-24.

[11] Esfand R and Tomalia DA: Poly(amidoamine) (PAMAM) dendrimers: from biomimicry to drug delivery and biomedical applications. Drug Discovery Today 2001; 6:427-36.

[12] Gupta U, Agashe HB , Asthana A and Jain NK: Dendrimers: novel polymeric nanoarchitectures for solubility enhancement. Biomacromolecules 2006; 7:649-58.

[13] Emanuele AD and Attwood D: Dendrimer-drug interactions. Advanced Drug Delivery Reviews 2005; 57:2147-62.

[14] Lee I, Athey BD, Wetzel AW: Structural Molecular Dynamics Studies on Polyamidoamine Dendrimers for a Therapeutic Application: Effects of $\mathrm{pH}$ and Generation. Macromolecules 2002; 35:4510-20.
(2) Priofect ${ }^{\mathrm{TM}}$, Priostar ${ }^{\mathrm{TM}}$ and Starburst for targeted diagnostic, therapeutic delivery for cancer cells

(3) SuperFect for Gene Transfection

(4) Stratus CS for Cardiac Marker

(5) Vivagel for preventing HIV

\section{CONCLUSION}

The possibility of medicinal and practical advances that dendrimers could lead to is endless. That possibility makes dendrimers a truly interesting topic of research. Recent success in simplifying and optimizing the synthesis of dendrimers provide a large variety of structures with reduced cost of their production. Although, as has been apparent by the many instances cited throughout this review where gaps in knowledge still remain and that must be plugged before dendrimers are ready for wide clinical use, their extreme versatility combined with the extensive research efforts now underway are sure to add sophistication to drugs already in use as well as spur the development of entirely new classes of therapeutics.

\section{Conflict of Interests}

The authors declare that there is no conflict of interests regarding the publication of this paper.

[15] Devarakonda B, Otto D and Judefeind A: Effect of $\mathrm{pH}$ on the solubility and release of furosemide from polyamidoamine (PAMAM) dendrimer complexes. Pharmaceutical Nanotechnology 2007; 345:142-53.

[16] Chai M, Niu Y and Youngs WJ: Structure and Conformation of DAB Dendrimers in Solution via Multidimensional NMR Techniques. Journal of the American Chemical Society 2001; 123:4670-78.

[17] Butler JE, Ni L, Nessler R: The physical and functional behaviour of capture antibodies adsorbed on polystyrene. Journal of Immunological Methods1992; 150:77-90.

[18] Ramzi A, Scherrenberg R and Joosten J: Structure-Property Relations in Dendritic Polyelectrolyte Solutions at Different Ionic Strength. Macromolecules 2002; 35:827.

[19] Topp A, Bauer BJ and Prosa TJ: Size Change of Dendrimers in Concentrated Solution. Macromolecules 1999; 32:8923-31.

[20] Caminade A, Laurent R and Majoral J: Characterization of dendrimers. Advance Drug Delivery Review 2005; 57:2130146.

[21] Sebastian RM, Blais JC and Caminade AM: Synthesis and photochemical behavior of phosphorus dendrimers containing azobenzene units within the branches and/ or on the surface. Chemistry European Journal 2002; 8:2172-83.

[22] Kim C and Son S: Preparation of double-layered dendritic carbosilanes. Journal of Organometallic Chemistry 2000; 599:123-27.

[23] Chu B and Hsiao BS: Small-angle X-ray scattering of polymers. Chemical Reviews 2001; 101:1727-62.

[24] Zeng F, Zimmerman SC and Kolotuchin SV: Supramolecular polymer chemistry: design, synthesis, characterization, and kinetics, thermodynamics, and fidelity of formation of selfassembled dendrimers. Tetrahedron 2002; 58:825-43.

[25] Francese G, Dunand FA, Loosli C: Functionalization of PAMAM dendrimers with nitronyl nitroxide radicals as models for the outer-sphere relaxation in dendritic potential MRI contrast agents. Magnetic Resonance Chemistry 2003; 41:81- 83.

[26] Kallos GJ, Tomalia DA, Hedstrand DM: Molecular weight determination of a polyamidoamine starburst polymer by electrospray-ionization mass Spectrometry. Rapid Communications in Mass Spectrometry 1991; 5:383-86.

[27] Trahasch B, Stu B, Frey H: Dielectric relaxation in carbosilane dendrimers with perfluorinated end groups. Macromolecules 1999; 32:1962-66. 
[28] Emanuele AD, Attwood D and Rmaileh RA: Dendrimers. Encyclopedia of Pharmaceutical Technology 2003; 3:1-21.

[29] Florence AT, Sakthivel T and Toth I: Oral uptake and translocation of a polylysine dendrimer with a lipid surface. Journal of Controlled Release 2000; 65:253-59.

[30] Pantzar N, Lundin S and Wester L: Bidirectional smallintestinal permeability in the rat to some common marker molecules in-vitro.Scandinavian Journal of Gastroenterology 1994; 29:703-9.

[31] Wiwattanapatapee R, Carreno-Gomez B, Malik N and Duncan R: PAMAM dendrimers as a potential oral drug delivery system: uptake by everted rat intestinal sacs in-vitro. Journal of Pharmacy and Pharmacology 1998; 50:99.

[32] Tripathi PK, Khopade AJ and Nagaich S: Dendrimer grafts for delivery of 5-fluorouracil. Pharmazie 2002; 57:261-64

[33] Wang ZX, Itoh Y and Hosaka Y: Novel transdermal drug delivery system with polyhydroxyalkanoate and starburst polyamidoamine dendrimer. Journal of Bioscience and Bioengineering 2003; 95:541-43.

[34] Z.X. Wang, I.Yoshiaki, H.Yoshifumi, "Mechanism of enhancement effect of dendrimer on transdermal drug permeation through polyhydroxyalkanoate matrix", Journal of Bioscience and Bioengineering, vol. 2003; 96:537-540.

[35] Cheng YY, Man N and Xu TW: Transdermal delivery of nonsteroidal anti-inflammatory drugs mediated by polyamidoamine (PAMAM) dendrimers. Journal of Pharmaceutical Sciences 2006; 96:595-602.

[36] Vandamme TF: Microemulsions as ocular drug delivery systems: Recent developments and future challenges. Progress in Retinal and Eye Research 2002; 21:15-34.

[37] Shimpi S, Chauhan B and Shimpi P: Cyclodextrins: Application in different routes of drug administration. Acta Pharmaceutica 2005; 55:139-56.

[38] Vandamme TF and Brobeck L: Poly (amidoamine) dendrimers as ophthalmic vehicles for ocular delivery of pilocarpine nitrate and tropicamide. J Control Release 2005; 102:23-38.

[39] Shaunak S, Thomas S and Gianasi E: Polyvalent dendrimer glucosamine conjugates prevent scar tissue formation. Nature Biotechnology 2004; 22:977-84.

[40] Duan X and SheardownH: Dendrimer crosslinked collagen as a corneal tissue engineering scaffold: Mechanical properties and corneal epithelial cell interactions. Biomaterials 2006; 27:4608-617.

[41] Yang W, Cheng Y and Xu T: Targeting cancer cells with biotindendrimer conjugates. European journal of medicinal chemistry 2009; 44:862-68.

[42] Zhuo RX, Du B and Lu ZR: In vitro release of 5-fluorouracil with cyclic core dendritic polymer. Journal of Controlled Release 1999; 57:249-57.

[43] Choi SK, Myc A and Silpe JE: Dendrimer-based multivalent vancomycin nanoplatform for targeting the drug-resistant bacterial surface. ACS Nano 2013; 7:214-28.

[44] Latallo K, Raczka JF and Quintana E: Intravascular and endobronchial DNA delivery to murine lung tissue using a novel, nonviral vector. Human Gene Therapy 2000; 11:3851395.
[45] Barbara K and Maria B: Review- Dendrimers: properties and applications. Acta Biochimica Polonica 2001; 48:99-208.

[46] Sonke S and Tomalia DA: Dendrimers in biomedical applications reflections on the Field. Advanced Drug Delivery Reviews 2005; 57:2106-29.

[47] Hecht S and Frechet JMJ: Dendritic encapsulation of function applying natures site isolation principle from biomimetics to materials science. Angewandte Chemie International Edition 2001; 40:74-91.

[48] Thayumanavan S, Bharathi P and Sivanandan K: Towards dendrimers as biomimetic macromolecules. Comptes rendus Chimie 2003; 6:767-78.

[49] Barth RF , Adams D and Soloway AH: Boronated starburst Dendrimer monoclonal antibody immunoconjugates: evaluation as a potential delivery system for neutron capture therapy. Bioconjugate Chemistry 1994; 5:58.

[50] Gillies ER and Fréchet JMJ: Dendrimers and dendritic polymers in drug delivery. Drug Discovery Today 2005; 10:3543.

[51] Tekade RK, Dutta T and Gajbhiye V: Exploring dendrimer towards dual drug delivery, $\mathrm{pH}$ responsive simultaneous drugrelease kinetics. Journal of Microencapsulation 2009; 26:28796.

[52] Kannan S, Dai H and Navath RS: Dendrimer-Based Postnatal Therapy for Neuroinflammation and Cerebral Palsy in a Rabbit Model. Science Translational Medicine 2012; 4:130.

[53] Sinan H and Battah: Synthesis and Biological Studies of 5Aminolevulinic Acid-Containing Dendrimers for Photodynamic Therapy. American Chemical Society: 2009.

[54] Luna BN, Godínez LA and Rodríguez FJ:Applications of Dendrimers in Drug Delivery Agents, Diagnosis, Therapy, and Detection. Journal of Nanomaterials, Hindawi Publishing Corporation 2014:19.

[55] Krause W, Schlichter HN and Maier FK: Dendrimers in diagnostics. Topics in Current Chemistry 2000; 210:261-308.

[56] Wiener EC, Brechbiel MW and Brothers H: Dendrimer-based metal chelates: a new class of magnetic resonance imaging contrast agents. Magnetic Resonance in Medicine 1994; 31:1-8.

[57] Svenson S and Tomalia DA: Dendrimers in biomedical applications- reflections on the field. Advanced Drug Delivery Reviews 2012; 64:102-15.

[58] Koten GV, Robert AG and Lucia A: Diaminoarylnickel(II) Pincer Complexes: Mechanistic Considerations in the Kharasch Addition Reaction, Controlled Polymerization, and Dendrimeric Transition Metal Catalysts. Accounts of Chemical Research 1998; 31:423-31.

[59] Howell BA and Fan D: Poly (amidoamine) dendrimersupported organoplatinum antitumour agents. Proceedings of the Royal SocietyA 2010; 466:1515-26.

[60] Klajnert B, Pikala $S$ and Bryszewska M: Haemolytic activity of polyamidoamine dendrimers and the protective role of human serum albumin. Proceedings of the Royal Society A 2010; 466:1527-34.

[61] Chauhan AS, Jain NK and Diwan PV: Pre-clinical and behavioural toxicity profile of PAMAM dendrimers in mice. Proceedings of the Royal Society A 2010; 466:1535-50. 